\title{
Therapeutic Plasmapheresis. A gate to an effective treatment of severe pathological conditions
}

\author{
Claudia Stefanutti ${ }^{1^{*}}$
}

1.Department of Molecular Medicine, "Sapienza" University of Rome, Immunohematology and Transfusion Medicine - Extracorporeal Therapeutic Techniques - Lipid Clinic and Atherosclerosis Prevention Centre -“Umberto I" Hospital, Rome, Italy (EU)

There are two ways to perform therapeutic plasmapheresis (TP). The simplest form is therapeutic plasma exchange (TPE) where the blood taken from the patient is separated in a centrifuge into cells and plasma. While the plasma is discarded, the cells together with a substitution fluid (e.g. fresh frozen plasma; albumin solution) are returned to the patient. The second form of treatment specifically removes the pathogenic substance alone by filtration on a specific substrate. A column is attached to the plasma line, selectively eliminating the pathogenic molecule and returning the patient's own plasma. Nowadays, practitioners of centrifugal TP tend to be hematologists and transfusionists, whilst the membrane-based approach appeals primarily to nephrologists or other specialists (experts in hemodialysis, surgeons). Adsorption, an apheresis technique conceivably with the highest selectivity, is founded on the principles of affinity chromatography in which plasma is passed through an insoluble matrix bound firmly to a ligand. The ligands could be either comparatively nonspecific chemical binders, e.g. heparin or charcoal, or specific recombinant protein antigens or monoclonal antibodies. A wide range of medical disorders of varying pathologies and end-organ involvement may be managed by TPE (1). TPE has still a crucial role in emergency. Critical care units staffed by highly trained medical and paramedical personnel using highly technical and sophisticated equipment might be supported by the provision of TPE. Timing is frequently of the essence in disorders in which plasma exchange (PEX) has a role, with decisions being based on a set of facts or clinical circumstances which may be rapidly changing (2) (Table 1). Severe Hypertriglyceridemia (sHTG) could be associated with acute pancreatitis (AP), a fatal and dreaded complication. Throughout the acute phase of hyperlipidemic pancreatitis (HLP), PEX (Lipid-apheresis) could be of considerable assistance not only in lowering TG

\section{Corresponding author:}

Professor Claudia Stefanutti, M.D., Ph.D.

Extracorporeal Therapeutic Techniques - Lipid Clinic and Atherosclerosis Prevention Centre - Immunohematology and Transfusion Medicine - Department of Molecular Medicine, University of Rome "Sapienza" - Umberto I Hospital-155, Viale del Policlinico, Rome, 00161, Italy (EU)

Email: claudia.stefanutti@uniroma1.it

Received: 2014-02-03| Accepted: 2014-02-20| Published:

2014-03-04 DOI: 10.7575/aiac.abcmed.14.02.02.01 


\begin{tabular}{cccc}
\hline Technique & Pathology & Emergency & Long-term \\
\hline Plasma-Exchange & Sepsis, MODS & $\mathbf{X}$ & \\
\hline Plasma-Exchange & $\begin{array}{c}\text { Hyperlipidemic } \\
\text { Pancreatitis } \\
\text { Hyperchylomicronemia }\end{array}$ & $\mathbf{X}$ & \\
\hline Plasma-Exchange & Familial Hypercholesterolemia & $\mathbf{X}$ \\
\hline $\begin{array}{c}\text { Lipoprotein Apheresis } \\
\text { (Dextran sulphate) }\end{array}$ & $\begin{array}{c}\text { Familial Hypercholesterolemia } \\
\text { Hyper Lp(a) lipoproteinemia }\end{array}$ & $\mathbf{X}$ \\
\hline $\begin{array}{c}\text { Lipoprotein Apheresis } \\
\text { (Dextran sulphate) }\end{array}$ & Preeclampsia & $\mathbf{X}$ & \\
\hline $\begin{array}{c}\text { Lipoprotein Apheresis } \\
\text { (H.E.L.P.) }\end{array}$ & $\begin{array}{c}\text { Familial Hypercholesterolemia } \\
\text { HyperLp(a)lipoproteinemia }\end{array}$ & $\mathbf{X}$ \\
\hline $\begin{array}{c}\text { Lipoprotein Apheresis } \\
\text { (H.E.L.P.) }\end{array}$ & Sepsis & $\mathbf{X}$ & \\
\hline
\end{tabular}

Table 1: Different clinical use of conventional and selective apheresis techniques in relation to the pathology to be treated in emergency or on long (chronic)-term.

levels but also in the prevention of recurrent HLP (3). Furthermore, patients with homo-, double- compound-, and heterozygous Familial Hypercholesterolemia and HyperLp(a) lipoproteinemia would benefit lipoproteinapheresis (LA) as an extracorporeal procedure providing selective removal of lipids and lipoproteins including Low Density Lipoproteins (LDL) and other apolipoprotein B100-containing lipoproteins $(4,5)$. Having numerous metabolic and clinical superiorities, LA characterizes an upgraded selective form of conventional extracorporeal therapies, e.g. plasma-exchange (PEX- Lipid-apheresis), which were broadlyused for managing severe hypercholesterolemia in the seventies. However, LA is primarily used in the treatment of previously-mentioned severe forms of dyslipidemia. FH patients are particularly prone to coronary ischemic events necessitating a tailored, intensive, efficient, continuous, and unceasing form of treatment. Obviously, a therapeutic approach exclusively relying on existing accessible medications would not lead to preferred clinical outcomes. The above reported clinical examples clearly suggest what differences exist between the non-selective and selective apheresis techniques as far as different pathologies exhibiting affinity are concerned. In particular, the clinical presentation, emergency or not, greatly affects the use of a given extracorporeal technique. However, depending upon the indication, a selective technique not usually utilized in emergency, such as selective dextran sulfate cellulose LA was recently suggested to treat acutely preeclampsia, where targeted therapies to stabilize the clinical manifestations and prolong pregnancy do not exist (6). A further complication of the apheresis issue is represented by the indication of sepsis with multi organ dysfunction syndrome (MODS) as the most common cause of death in patients in non-coronary intensive care units. Presently, an effective treatment to reduce mortality in sepsis and MODS patients is still not available. Therapeutic PEX in the management of sepsis and MODS was suggested several years ago and more recently $(7,8)$. On the other side, even a selective LA technique such as Heparin induced Extracorporeal Lipoprotein Precipitation (H.E.L.P.) was used in the treatment of sepsis and it is still on use (9). In conclusion, extracorporeal therapeutic techniques can be 
clinically helpful in emergency, in deferred urgency and in chronic, long-term use. As these techniques are invasive and relatively complex, appropriate equipment and highly skillful medical and non-medical staff personnel is a necessary complement.

\section{Key words:}

Therapeutic Plasmapheresis, Plasma Exchange, Lipoprotein Apheresis, Hyperlipidemic
Pancreatitis, Acute Pancreatitis, HomozygousHeterozygous Familial Hypercholesterolemia, Hyper Lp(a) lipoproteinemia

\section{Conflict of interest statement}

The author have no conflict of interest to declare.

\section{References}

1. Schwartz J, Winters JL, Padmanabhan A, Balogun RA, Delaney M, Linenberger ML, Szczepiorkowski ZM, Williams $\mathrm{ME}, \mathrm{Wu} \mathrm{Y}$, Shaz BH. Guidelines on the use of therapeutic apheresis in clinical practice-evidence-based approach from the Writing Committee of the American Society for Apheresis: the sixth special issue. J Clin Apher. 2013; 28(3):145-284.

2. Stefanutti C, Di Giacomo S, Labbadia G. Timing clinical events in the treatment of pancreatitis and hypertriglyceridemia with therapeutic plasmapheresis. Transfus Apher Sci. 2011; 45(1):3-7.

3. Stefanutti C, Labbadia G, Morozzi C. Severe hypertriglyceridemia-related acute pancreatitis. Ther Apher Dial. 2013; $17(2): 130-7$.

4. Thompson GR. The evidence-base for the efficacy of lipoprotein apheresis in combating cardiovascular disease. Atheroscler Suppl. 2013; 14(1):67-70.

5. Stefanutti C, Julius U.Lipoprotein apheresis: state of the art and novelties. Atheroscler Suppl. 2013; 14(1):19-27.

6. Thadhani R, Kisner T, Hagmann H, Bossung V, Noack S, Schaarschmidt W, Jank A, Kribs A, Cornely OA, Kreyssig C, Hemphill L, Rigby AC, Khedkar S, Lindner TH, Mallmann P, Stepan H, Karumanchi SA, Benzing T. Pilot study of extracorporeal removal of soluble fms-like tyrosine kinase 1 in preeclampsia. Circulation. 2011; 124(8):940-50.

7. De Simone N, Racsa L, Bevan S, Matevosyan K, Valley T, Girod C, Sarode R Therapeutic plasma exchange in the management of sepsis and multiple organ dysfunction syndrome: A report of three cases. J Clin Apher. 2013 Sep 2. doi: 10.1002/jca.21296.

8. Busund R, Koukline V, Utrobin U, Nedashkovsky E. Plasmapheresis in severe sepsis and septic shock: a prospective, randomised, controlled trial. Intensive Care Med 2002; 28: 1434-143.

9. Samtleben W, Bengsch S, Boos KS, Seidel D. HELP apheresis in the treatment of sepsis. Artif Organs. 1998; 22(1):43-6. 